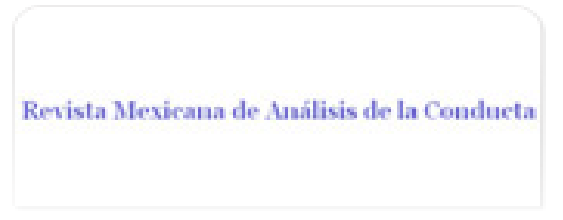

Revista Mexicana de Análisis de la Conducta ISSN: 0185-4534

editora@rmac-mx.org

Sociedad Mexicana de Análisis de la Conducta México

Vite Sierra, Ariel; López Rodríguez, Florente LA ASINCRONÍA MATERNA AUTORITARIA Y PERMISIVA EN EL MALTRATO INFANTIL Revista Mexicana de Análisis de la Conducta, vol. 36, núm. 3, diciembre-marzo, 2010, pp. 23-34 Sociedad Mexicana de Análisis de la Conducta Guadalajara, México

Disponible en: http://www.redalyc.org/articulo.oa?id=59315690002

Cómo citar el artículo

- Número completo

- Más información del artículo

Página de la revista en redalyc.org

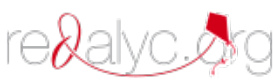

Sistema de Información Científica Red de Revistas Científicas de América Latina, el Caribe, España y Portugal Proyecto académico sin fines de lucro, desarrollado bajo la iniciativa de acceso abierto 


\title{
LA ASINCRONÍA MATERNA AUTORITARIA Y PERMISIVA EN EL MALTRATO INFANTIL
}

\author{
THE MOTHERING AUTHORITARIAN AND PERMISSIVE ASYNCHRONY \\ IN CHILD ABUSE \\ ARIEL VITE SIERRA Y FLORENTE LÓPEZ RODRÍGUEZ \\ Universidad Nacional Autónoma de México, Facultad de Psicología
}

\begin{abstract}
Resumen
El objetivo del estudio fue comparar dos formas de asincronía materna: autoritaria y permisiva, entre díadas con historia de maltrato físico y díadas control. Participaron 17 díadas madre-hijo con historia de maltrato físico y 17 díadas sin historia de maltrato. Las edades de los niños oscilaron entre cuatro y nueve años, con una edad media de 6.8 años. Ambos grupos de díadas se observaron en 3 sesiones de 30 minutos cada una bajo una condición académica. Los resultados indican que las madres maltratadoras fueron más autoritarias y permisivas que las madres control. La discusión se centra en los tipos de asincronía materna y las diferencias entre las características de ambos grupos que pueden dar cuenta de los estilos paternales de cada tipo de muestra.

Palabras clave: maltrato físico, asincronía autoritaria, asincronía permisiva, indiscriminación materna, interacción social

Proyecto financiado por UNAM DGAPA IN 302207. Ambos autores contribuyeron de manera equiparable en la recolección y análisis de datos, y en la preparación del manuscrito. La obtención de recursos corrió a cargo de Ariel Vite. Dirigir la correspondencia a Ariel Vite Sierra, Facultad de Psicología, UNAM. Av. Universidad 3004, Col. Copilco-Universidad, CP 04510, Coyoacán, México, tel. +52(55) 56220555 ext.41244, correo-e: avite@servidor.unam.mx
\end{abstract}




\begin{abstract}
The objective of the present study was to compare two forms of maternal asynchrony: authoritarian and permissive between dyads with history of physical abuse and control dyads. Participated 17 mother-infant dyads with a history of physical abuse and 17 dyads without a history of abuse. The children's ages ranged between four and nine years with a mean age of 6.8 years. Both groups of dyads were observed in 3 sessions of 30 minutes in an academic condition. The discussion focuses on the types of maternal asynchrony and differences between the characteristics of both groups that may account for the parenting styles of each type of sample.
\end{abstract}

Key Words: Physical abuse, authoritarian asynchrony, permissive asynchrony, social interaction.

El maltrato infantil representa un problema mundial con graves consecuencias en la salud física, psicológica, el bienestar de las víctimas, y por extensión a la sociedad. Al respecto en 1999 la Organización Mundial de la Salud estimó que 40 millones de niños entre 0 y 14 años de edad sufrían maltrato o negligencia en el mundo (Clark \& Clark, 2001). En México, de acuerdo a la Dirección General del Servicio Público de Localización Telefónica (Locatel) de enero a diciembre de 2008 se reportaron 1,298 casos de maltrato, 656 niñas y 642 niños de 1 a 17 años. En el período comprendido de enero a marzo del 2009 se reportaron 247 casos, 108 niñas y 139 niños. Por lo que concierne a los agresores, se señala que la madre aparece como el principal agresor en el $92.5 \%$ de los casos, la madrasta en el $1.6 \%$, la abuela en el 2.6 y en el restante $3.3 \%$ están involucrados el tío, los primos, padrastro y hermanos (Locatel, 2009). Gran parte de los agresores son personas jóvenes, entre 25 y 30 años de edad, sin diferencias en la clase social (Kitsu, 1991). Como se observa en las estadísticas, el principal miembro de la familia en generar maltrato físico es la madre.

Diversos trabajos empíricos muestran la importancia que tiene la maternidad en la formación de la conducta social del niño, y ha sido el centro de atención para el estudio de los problemas de comportamiento y maltrato infantil (Azar, Reitz \&. Goslin, 2008; Ison \& Morelato, 2008; Patterson, 2002).

Estos estudios señalan que la maternidad comprende aspectos tales como afecto, disciplina, enseñanza, juego, escuchar y observar, los cuales se articulan en relación al amplio rango de la conducta infantil. Esta articulación es primordial, porque el arreglo contingente de estos eventos en relación a la conducta infantil es más importante que la distribución de las conductas maternales a través del tiempo (Bretherton \& Waters, 1985; Maccoby \& Martin, 1983). Cuando la articulación falla y la madre y el niño quedan "fuera de sincronía", aumenta la probabilidad de que se involucren en disputas que pueden generar el proceso de escalamiento y finalizar en violencia física en contra del niño (Cerezo \& Pons-Salvador, 1996; Patterson, 2002; Patterson, Reid, \& Dishion, 1992). 
La sincronía es un concepto de la interacción social que hace referencia a la continuidad de las acciones y reacciones apropiadas en una relación diádica, en este caso madre-hijo. Las dos palabras adecuadas y continuidad definen la sincronía interaccional y también distinguen la capacidad dual de la madre para reaccionar tanto a respuestas específicas como a patrones de respuesta de su hijo (Whaler \& Smith, 1999).

Por lo tanto, cuando una madre presenta un estilo asincrónico, sus acciones y reacciones resultan inoportunas, inconsistentes e inapropiadas respecto al amplio repertorio del comportamiento infantil. El estilo maternal asincrónico engloba dos prácticas caracterizadas como autoritarismo y permisividad (Baumrind, 1983). La primera de ellas implica demandas rígidas para obtener la obediencia infantil, y la segunda se refiere a la laxitud maternal, pero ambas tienden a generar un proceso de coerción (Lahey, Conger, Atkeson, \& Treiber, 1984). Las madres que manifiestan un estilo autoritario son propensas a emplear tácticas aversivas $y$, dado este sesgo, sus acciones correctivas representan un desafío a la independencia del niño y predisponen su conducta aversiva. Por otro lado, las madres permisivas, tienden a alentar todas las conductas de sus niños, sin considerar el valor de ajuste social que pueden tener para sus hijos.

Si las relaciones asincrónicas entre madre-hijo genera un sesgo autoritario o permisivo, ambas formas de inconsistencia pueden propiciar tanto problemas de antisocial como episodios de maltrato físico (Shaw \& Bell, 1993). En virtud de que los factores vinculados con el maltrato físico al menor son el centro de interés del presente estudio, la comparación entre estos sesgos resulta pertinente para analizar el grado en que estos dos estilos paternales desadaptativos se asocian al maltrato físico infantil.

La asincronía autoritaria y permisiva fue definida de manera operacional por Wahler y Dumas (1986) de acuerdo a un modelo molecular de crianza desadaptada, siendo la primera conceptuada como las reacciones aversivas inoportunas de la madre en relación al comportamiento prosocial infantil, y la segunda como las reacciones positivas inoportunas de la madre en relación al comportamiento prosocial y aversivo infantil. Lo cual ha mostrado que covaría con el comportamiento infantil inapropiado (Cerezo \& D'Ocon, 1999; Wahler, Williams \& Cerezo, 1990).

En resumen, cuando el estilo de una madre es asíncrono, sus reacciones son inoportunas, generando como resultado que sus diversas respuestas sean confusas e inapropiadas con respecto al amplio rango de respuestas de sus hijos. Dentro de este desajuste diádico, los patrones coercitivos de madre-hijo pueden irrumpir y convertirse en la base social para el maltrato físico infantil (Cerezo \& D’Ocon, 1999; Vite \& López, 2007). Sin embargo, no existe suficiente evidencia respecto al grado en que estos estilos de crianza desadaptativa están asociados con el maltrato infantil, por lo que resulta relevante llevar a cabo las comparaciones pertinentes. Con base en lo señalado, el objetivo del estudio fue comparar las dos formas de asincronía materna, autoritaria y permisiva entre díadas con historia de maltrato físico y díadas control. 


\section{Participantes}

Participaron un total de 34 díadas madre-hijo: 17 con historia de maltrato físico y 17 controles. Las díadas con historia de maltrato físico fueron canalizadas al Centro de Servicios Psicológicos de la Facultad de Psicología de la UNAM por presentar historia de violencia al menor, lo cual fue confirmado por los resultados obtenidos con la Escala para la Detección de Madres Maltratadoras Física y/o Emocionalmente (Pérez, 2006), en virtud de que el valor obtenido fue de 74 , que se estima como un tipo de maltrato físico medio. Las madres de este grupo tenían edades comprendidas entre 21 y 47 años $(M=38.07)$ con un nivel socioeconómico medio. El $90 \%$ eran madres casadas y el $75 \%$ trabajaban. Los niños de esta muestra fueron cinco niñas y 12 niños con un rango de edad entre los seis y los siete años, con una media de 6.2 años. Las díadas control, sin historia de maltrato, se seleccionaron de una escuela primaria oficial ubicada en el sur de la ciudad de México. El resultado derivado de la Escala para la Detección de Madres Maltratadoras Física y/o Emocionalmente fue de 30 (DE= 21), que se considera como ausencia de maltrato físico. Las edades de las madres oscilaron entre los 26 y los 45 años $(M=36.14)$, con un nivel socioeconómico medio, $95 \%$ eran casadas, el $80 \%$ trabajaba. Los niños de esta muestra fueron cinco niñas y 12 niños con un rango entre los seis y los siete años de edad, con una media de 6.5 años. Los grupos de díadas se igualaron con respecto a la edad, sexo y escolaridad de los niños.

\section{Instrumentos y variables}

Para evaluar las conductas de interés se utilizó el Sistema Observacional de Interacción (SOI; Vite, García \& Rosas, 2006), el cual es un sistema computarizado de registro observacional para el estudio de la interacción madre-niño en ambientes controlados, y comprende un conjunto de categorías de comportamiento, mutuamente excluyentes y exhaustivas, de la madre y del niño (ver Anexo 1). Sus principales características son: a) permite un registro continuo de las interacciones, b) posibilita la codificación secuencial de eventos, c) permite la obtención de medidas de frecuencia por sesión de cada código, tanto de la madre como del hijo y d) permite derivar tasas de respuesta por minuto.

\section{Codificación}

Las categorías obtenidas mediante el SOI se codificaron de acuerdo a los siguientes agrupamientos:

Maternas.

Aversiva. Comprendió las conductas de amenazar, desaprobar y regañar. Prosocial. Abarcó las conductas de aproximación social, aprobar y obedecer. Instruccional. Incluyó verbalizaciones concisas y claras que señalen el cómo, cuándo 
y dónde se tiene que realizar una conducta.

Neutral. Comprendió la conducta de Supervisar.

Infantiles.

Prosocial. Incluyó las conductas de obedecer y realizar la actividad.

Aversiva. Comprendió las conductas de desobedecer y repelar. (Ver anexo 1 para la definición de las conductas).

Asimismo, se evaluaron de la siguiente manera los dos tipos de asincronía materna:

a. Asincronía autoritaria se definió como la suma de episodios de observación de las conductas maternas aversivas contingentes a la conducta infantil prosocial.

b. Asincronía permisiva se definió como la suma de episodios de observación de las conductas maternas prosociales contingentes a la conducta infantil aversiva

\section{Procedimiento}

Los dos grupos se observaron en escenarios diferentes. El grupo de maltrato fue evaluado en una cámara de Gessell del Centro de Servicios Psicológicos de la Facultad de Psicología de la UNAM, mientras que las díadas control fueron evaluadas en la sala de sus hogares. Sin embargo, las condiciones de observación fueron las mismas: se programó una actividad académica (tarea), supervisada por la madre de acuerdo al nivel escolar de cada niño.

Se llevaron a cabo tres sesiones de 20 minutos cada una, con lo cual se obtuvo un total 32 horas de observación del comportamiento diádico. Los observadores fueron estudiantes universitarios, capacitados en el uso del SOI-I.

\section{Resultados}

Concordancia entre observadores

Se obtuvo un índice de concordancia entre observadores contrastando los dos registros de los catálogos conductuales obtenidos por los observadores independientes. Para ello se eligieron al azar un 30\% del total de sesiones de observación tanto de la madre como del niño. El índice de concordancia entre observadores se obtuvo a través del Coeficiente Kappa de Cohen (Bakeman \& Gottman, 1989). Los valores Kappa resultantes se muestran en la Tabla 1, en donde se puede apreciar que oscilaron entre .80 y .86, por lo que pueden ser considerados como "excelentes" de acuerdo a la clasificación propuesta por Fleiss (1981). 
Tabla 1.

Índice de concordancia obtenido en la codificación de las conductas materna e infantil de los grupos control y maltrato

\begin{tabular}{|c|c|c|}
\hline & Grupo Maltrato & Grupo Control \\
\hline Conducta maternal prosocial & .82 & .82 \\
\hline Conducta maternal aversiva & .80 & .82 \\
\hline Conducta infantil prosocial & .86 & .82 \\
\hline Conducta infantil Aversiva & .88 & .86 \\
\hline
\end{tabular}

Comparaciones de grupo

Los principales análisis se enfocaron en las mediciones de las conductas prosocial y aversiva, tanto de las madres como de los niños, así como los dos tipos de asincronía. A fin de realizar las comparaciones entre grupos, se empleó la prueba no paramétrica U de Mann-Whitney. En la Tabla 2 se presentan el porcentaje promedio de las diversas categorías de comportamiento materno. No se obtuvieron diferencias estadísticamente significativas entre grupos en ninguna de las categorías de comportamiento.

Tabla 2.

Frecuencias promedio de las conductas maternas prosociales y aversivas en los grupos Maltrato y Control

\begin{tabular}{lll}
\hline Conductas & Grupo Maltrato & Grupo Control \\
\hline Prosociales & 44 & 41 \\
Aversivas & 2 & 3 \\
\hline
\end{tabular}

En la Tabla 3, se muestran las frecuencias promedio del comportamiento infantil. De las comparaciones posibles, solo se obtuvieron diferencias significativas en el comportamiento aversivo entre grupo control y grupo de niños maltratados ( $Z$ $=14.96, \mathrm{p}<0.0001$ ), es decir los niños del grupo control observan mayores tasas de conducta desobediente y de repelar que los niños maltratados.

Tabla 3.

Frecuencias promedio de las conductas infantiles prosociales y aversivas en los grupos Maltrato y Control

\begin{tabular}{lll}
\hline Conductas & Grupo Maltrato & Grupo Control \\
\hline Prosociales & 84 & 80 \\
Aversivas & .5 & $3^{*}$ \\
\hline
\end{tabular}


Asincronía Autoritaria. En la Tabla 4 se aprecian las diferencias en los episodios de asincronía maternal autoritaria entre los grupos de maltrato y control. Como se observa en la Tabla, las madres maltratadoras son más propensas a reaccionar de manera aversiva al comportamiento infantil aversivo, en comparación con su grupo control (madres normales), lo cual fue confirmado por la comparación estadística de las frecuencias promedio $(Z=32.13, p<0.001)$.

Tabla 4.

Porcentaje de episodios de asincronía autoritaria en los dos grupos de díadas

\begin{tabular}{llll}
\hline Grupos & Episodios & $\mathrm{Z}$ & $\mathrm{P}$ \\
\hline Maltrato & 74 & 32.13 & 0.001 \\
Control & 15 & & \\
\hline
\end{tabular}

Asincronía Permisiva. En la Tabla 5 se presentan las diferencias en los episodios de asincronía permisiva materna. En donde se observa que las madres maltratadoras muestran mayor probabilidad de utilizar atención positiva indiscriminada que las madres del grupo control $(Z=23.56, p<0.0001)$.

Tabla 5.

Porcentaje de episodios de asincronía permisiva en ambos grupos de díadas.

\begin{tabular}{llll}
\hline Grupos & Episodios & $\mathrm{Z}$ & $\mathrm{P}$ \\
\hline Maltrato & 58 & 23.56 & 0.00 \\
Control & 44 & & \\
\hline
\end{tabular}

\section{Discusión}

El propósito del estudio fue comparar dos formas de asincronía: autoritaria y permisiva entre díadas con historia de maltrato físico y díadas control. Con este fin se programó una condición de observación de alta demanda: realización de la tarea escolar por parte de los niños.

En general, los resultados señalan que la asincronía materna y el maltrato físico infantil están relacionados. Al respecto, las madres maltratadoras generaron tasas más altas de ambas formas de asincronía, en contraste con las madres control que fueron más proclives a generar asincronía permisiva. De manera particular, la asincronía significa que las madres maltratadoras no son sensibles a la conducta prosocial de sus hijos, respondiendo de aversiva. En segundo lugar, significa que su empleo generalizado de reacciones aversivas, además de no promover la conducta prosocial de sus hijos crea incertidumbre en ellos. 
Este tipo de patrón interactivo asincrónico es una característica de la relación padre-hijo en niños con problemas de conducta en edad escolar (Patterson, 1976; Wahler \& Dumas, 1986). Bajo este contexto, cuando el niño se comporta de forma prosocial, positiva o neutra, es muy probable que la madre se comporte de forma impredecible, generándose el fenómeno de asincronía. Sin embargo, si la conducta del niño es aversiva, la probabilidad de una respuesta materna más discriminada y consistente se incrementa. Se ha demostrado que la impredictibilidad actúa como estímulo aversivo, tanto en estudios con animales como con humanos, y que cualquier conducta que permita al individuo escapar de esta asincronía será reforzada negativamente (Patterson, 1982, Wahler \& Dumas, 1986). Por consiguiente, es posible que la relación asincrónica propicie la conducta aversiva del niño.

Este fenómeno ha sido referido como "escape de la incertidumbre", y ha sido observado en díadas con historia de maltrato infantil (Cerezo \& D’Ocon, 1999), en donde el niño emplea conductas aversivas en sus interacciones con su madre a fin de sustituir la conducta paternal impredecible por conducta predecible. Bajo estas circunstancias, la aversividad infantil es reforzada negativamente como resultado de reducir la impredictibilidad en el ambiente. Esta situación puede explicar los hallazgos paradójicos que indican que, para algunos niños, el castigo incrementa la tasa de agresión en vez de disminuirla.

En este sentido, cuando las madres maltratadoras hacen uso excesivo de las atenciones aversivas, no solo fracasan en estimular la conducta prosocial infantil, sino que también crean incertidumbre en ellos. Bajo estas condiciones sociales, los niños podrían responder con comportamiento aversivo como el único medio viable para obtener previsibilidad (predictibilidad) y tal vez el cumplimiento por parte de las madres de sus demandas (Cerezo \& D’Ocón, 1999; Vite \& López, 2007).

Asimismo, se observó que las madres maltratadoras reaccionaban de manera prosocial ante el comportamiento infantil aversivo, lo que sugiere que la reducción de este tipo de conducta materna funciona como reforzador negativo de la respuesta infantil aversiva (Cerezo, D`Ocon \& Doltz, 1996; Vite \& López, 2007).

Por otra parte, ambos tipos de asincronía se manifestaron, a pesar de que el comportamiento infantil de los niños maltratados fue igual de prosocial y menos aversivo que el de sus controles "normales".

En conclusión, las madres con un estilo asincrónico autoritario parecen propensas a utilizar tácticas aversivas en la crianza de sus hijos, y a causa de esta tendencia, sus acciones correctivas desafían la independencia y promueven el comportamiento aversivo de sus hijos. Por otro lado, las madres permisivas se muestran sesgadas en su perspectiva positiva, lo que fomenta todo tipo de comportamiento de sus hijos, independiente del valor o importancia que puedan tener en el desarrollo de los niños.

Un punto importante en estos resultados, tanto por su alcance teórico como práctico, es el hecho de que las madres maltratadoras actúan de forma inconsistente con sus hijos. Una maternidad adecuada requiere sensibilidad a las necesidades y capacidades del niño para solicitar determinadas conductas y proporcionar las consecuen- 
cias apropiadas según las expectativas que se tengan del niño, las cuales deben ser realistas y ajustadas a su nivel de desarrollo.

Tomando en cuenta que los hallazgos obtenidos señalan la pertinencia e importancia de los estudios observacionales para la investigación de la asincronía en el maltrato infantil, se considera oportuno en estudios subsecuentes incorporar variables maternas tales como depresión, estrategias de solución de problemas, control de ira y apoyo social, que son frecuentemente asociados con el esta problemática, y asimismo, llevar a cabo las sesiones de observación en un mismo escenario, a fin de que la variabilidad en las interacciones madre-hijo sea una posible explicación a los diferentes patrones interactivos madre-hijo.

Finalmente, se puede suponer la posibilidad de que las diferencias encontradas en este estudio entre diadas con historia de maltrato físico y control no sean exclusivas de estos grupos. En otras palabras, es posible que otros grupos con problemáticas clínica diferentes pudieran estar asociados con un estilo de crianza similar. Futuros estudios con diadas con diversas problemáticas podrían arrojar más luz sobre la relación entre los tipos de asincronía y el maltrato infantil

\section{Referencias}

Azar, S. T., Reitz, E. B., \&. Goslin, M. C (2008). Mothering: Thinking is part of the job description: Application of cognitive views to understanding maladaptive parenting and doing intervention and prevention work. Journal of Applied Developmental Psychology, 29, 295-304.

Baumrind, D. (1983). Rejoinder to Lewis' reinterpretation of parental firm control effects: Are authoritative families really harmonious? Psychological Bulletin, 94, 132-142.

Bakeman, J., \& Gottman, R. (1989). Observación de la interacción: Introducción al análisis secuencial. Madrid: Morata.

Bretherton, I., \& Waters, E. (Eds.). (1985). Growing points of attachment theory and research. Monographs of the Society for Research in Child Development, 50 (1-2, Serial N. 209).

Cerezo, M.A., \& Pons-Salvador, G. (1996). Ecosystem adversity as setting factors in mother's perception of child behavior and indiscriminate mothering. European Journal of Psychological Assessment, 12, 103-11.

Cerezo, M. A., D’Ocon, A., \& Doltz, L. (1996). Mother-child interactive patterns in abusive families versus nonabusive families: An observational study. Child Abuse and Neglect, 20, 573-587.

Cerezo, M. A., \& D’Ocon, A. (1999). Sequential analysis in coercitive mother-child interaction pattern in maltreated children. Child Abuse and Neglect, 2, 99-113.

Cerezo, M. A., \& Dolz, L. (2000). Análisis de datos. Medidas/Data Analysis. Measurements. In M. A. Cerezo, (Ed.), Interacción familiar/Family Interaction- SOC III. (CD-ROM format). Valencia: Publicaciones de la Universidad de Valencia. 
Clark, R. E., \& Clark, J. F. (2001). The Encyclopedia of Child Abuse, 2a. Ed. Nueva York: Facts On File, Inc.

Fleiss, J. (1981). Statistical Methods for Rates and Proportions. New York. 2nd ed. p. 321.

Ison, M.S. \& Morelato, G.S. (2008). Habilidades socio-cognitivas en niños con conductas disruptivas y víctimas de maltrato. Universitas Psychologica, 7, 357-37.

Kitsu, M. (1991). El niño maltratado. En Memorias del Primer Seminario Interdisciplinario e Internacional: El maltrato al niño y sus Repercusiones Educativas. México.

Lahey, B.B., Conger, R., Atkeson, B.M., \& Treiber, F.A. (1984). Parenting behavior and emotional status of physically abusive mothers. Journal of Consulting and Clinical Psychology, 36, 252-256.

Maccoby, E.E., \& Martin, J.A. (1983). Socialization in the context of the family: Parent-child interaction. In P.H. Mussen (Series Ed.) \& E.M. Hetherington (Vol. Ed.), Handbook of Child Psychology: Vol. 4. Socialization. Personality and social development (pp. 1-102). New York: Wiley.

Patterson, G.R. (1976). The aggressive child: Victim and architect of a coercive system. In E.J. Mash,L.A. Hamerlynck, \& L.C. Handy (Eds.), Behavior modification and families (pp. 32-50). New York: Brunner/Mazel.

Patterson, G.R. (1982). Coercive family process. Eugene, OR: Castalia

Patterson, G.R., Reid, J.B., \& Dishion, T.J. (1992). A social learning approach: Antisocial boys. Eugene, OR: Castalia.

Patterson, G. R. (2002). Etiology and treatment of child and adolescent antisocial behavior. The Behavior Analyst Today, 3, 133-144.

Pérez, J. R. (2006). Escala para la Detección de Madres Maltratadoras Física y/o Emocionalmente. Documento Inédito. Facultad de Psicología, UNAM.

Servicio Público de Localización Telefónica (Locatel). (2009) http://www.locatel.df.gob.mx/

Shaw, D.S., \& Bell, R.Q. (1993). Developmental theories of parental contributors to antisocial behavior. Journal of Abnormal Child Psychology, 21, 493-518.

Strand, P. (2000). A modern behavioral perspective on child conducts disorders: Integrating behavioral momentum and matching theory. Clinical Psychology Review, 20, 593-615.

Vite, A., García, R., \& Rosas, C. (2006). Sistema Observacional de Datos. SOI-I. Facultad de Psicología, UNAM.

Vite, A., \& López, F. (2007). La regulación aversiva en las interacciones madre-niño en díadas con historia de maltrato infantil. Apuntes de Psicología, 25, 145-156.

Wahler, R.G., \& Dumas, J.E. (1986). Maintenance factors in coercive mother-child interactions: The compliance and predictability hypotheses. Journal of Applied Behavior Analysis, 19, 13-22.

Whaler, R. G., \& Smith, G. D. (1999). Effective parenting as the integration of lessons and dialogue. Journal of Child and Family Studies, 8, 135-149.

Whaler, R., G., Williams, A. J., \& Cerezo, M. A. (1990). The compliance and predictability hypothesis: Sequential and correlational analyses of coercitive motherchild interactions. Behavioral Assessment, 12, 391-407. 
Maltrato infantil

\section{Anexo 1}

Conductas de la madre

1) Atención social y/o aproximación (AS): Esta categoría se codifica para cualquier tipo de contacto físico o verbal de la madre hacia el niño.

2) Instrucciones (in): Verbalizaciones concisas y claras que indiquen la conducta a realizar.

3) Obedecer (ob): Realizar una acción en relación a una petición o solicitud del niño

4) Rehusarse (ru): No realizar acción alguna ante una petición o solicitud del niño.

5) Amenazar (am): Verbalizaciones que impliquen consecuencias aversivas por no realizar una conducta.

6) Desaprobar (ds): Verbalizaciones y/o movimientos horizontales de cabeza para condenar la conducta del menor.

7) Regañar: Verbalizaciones en tono de voz alto, para expresar disgusto o enojo hacia la conducta del niño.

8) Aprobación (ap): Reconocimientos verbales positivos al niño, los cuales no son referentes a la conducta directa que el niño está realizando.

9) Supervisar (sup): Observar la actividad del niño, sin proporcionar ningún tipo de instigación física y/o verbal.

10) Otras: Cualquier conducta no contemplada en las anteriores 
Anexo 1 (cont.)

Conductas del niño

(1) Atención y/o Proximidad Social (AP): Cualquier contacto físico o verbal del niño hacia su madre

2) Obedecer (ob): Llevar a cabo la instrucción dada por la madre.

3) Desobedecer (de): No ejecutar las instrucciones proporcionadas por la madre.

4) Petición (pe): Solicitudes directas o indirectas dirigidas a la madre, las cuales debe designar un referente o clase de referente evidente (Pedir un libro, cuaderno, ayuda). 5) Repelar (re): Verbalizaciones que señalan contraposición a las instrucciones de la madre.

6) Quejarse (qe): Verbalizaciones que justifican la conducta infantil para no realizar la instrucción materna. (Señalando cansancio, fatigo, que lo hará más tarde, o que la petición es redundante o que no puede realizarla). (Verbalizaciones que indiquen los impedimentos que tiene el niño para realizar alguna actividad)

7) Realizar la Actividad (ra): Involucrarse de manera directa en la tarea en cuestión.

8) Otras (ot): Cualquier conducta no contemplada en las anteriores. 